\title{
Epidemiologia da Demência e da Doença de Alzheimer em Portugal: Estimativas da Prevalência e dos Encargos Financeiros com a Medicação
}

\author{
The Epidemiology of Dementia and Alzheimer Disease in Portugal: \\ Estimations of Prevalence and Treatment-Costs
}

Isabel SANTANA ${ }^{1,2,3}$, Filipa FARINHA ${ }^{4}$, Sandra FREITAS ${ }^{1,5}$, Vítor RODRIGUES ${ }^{6}$, Álvaro CARVALHO ${ }^{7,8}$

Acta Med Port 2015 Mar-Apr;28(2):182-188

\section{RESUMO}

Introdução: A incidência e prevalência de demência e de Doença de Alzheimer aumentam com a idade, duplicando a cada cinco anos após a sexta década de vida. Portugal é um país envelhecido, previsivelmente com um número crescente de casos de demência. No entanto, os dados epidemiológicos são escassos e os estudos sobre os custos da doença praticamente inexistentes. Propomo-nos apresentar uma estimativa actualizada da prevalência de demência/ Doença de Alzheimer em Portugal e inferir, a partir da prescrição específica para demência, o número de diagnósticos efectivos e os encargos financeiros com esses medicamentos.

Material e Métodos: À população residente em Portugal (2013), aplicámos os valores de prevalência de demência para a Europa Ocidental (estudo da Alzheimer's Disease International). A estimativa dos diagnósticos efectivos de Doença de Alzheimer e dos encargos financeiros com medicação específica baseou-se nas informações do Intercontinental Marketing Services Health (IMSH) - 2013. Resultados: O número estimado de Portugueses com mais de 60 anos e com demência foi 160287, o que corresponde a 5,91\% deste universo populacional. Sabendo que a Doença de Alzheimer representa 50-70\% dos casos, inferimos que existirão entre 80144 e 112201 doentes. Por outro lado, os dados da IMSH indicam que estarão diagnosticados e a receceber anti-demenciais 76250 doentes, representando um encargo financeiro de $37 \mathrm{M} € /$ ano.

Conclusão: O envelhecimento da população incrementa o número de casos de demência. Aparentemente, nem todos os doentes com Doença de Alzheimer recebem a medicação aconselhada, sugerindo que esta condição ainda está sub-diagnosticada. A evolução tem sido positiva, com incremento do número de doentes tratados e redução dos custos com fármacos específicos.

Palavras-chave: Custos do Tratamento; Demência; Doença de Alzheimer; Portugal; Prevalência.

\section{ABSTRACT}

Introduction: The incidence and prevalence of global dementia and Alzheimer's disease (AD) increase with age, almost doubling every five years after the sixth decade of life. Demographic aging is a reality in Portugal, being expectable that the number of dementia cases also increases. Even so, dementia-epidemiological data in Portugal is scarce and cost-of-illness studies are almost inexistent.

Our aims were to obtain up-to-date information about the prevalence of dementia/ Alzheimer's disease in Portugal, to estimate the number of cases effectively diagnosed as Alzheimer's disease and to determine illness-costs with specific dementia treatment.

Material and Methods: The numbers of age-adjusted prevalence of dementia obtained for Occidental Europe (Alzheimer's Disease International study), where applied to the resident population in Portugal (2013). Estimations related to diagnosis and treatment-costs were based in data provided by the Intercontinental Marketing Services Health (IMSH) - 2013.

Results: The estimated number of Portuguese people with dementia among those aged $\geq 60$ years, is 160287 , representing $5.91 \%$ of this population-stratum. Knowing Alzheimer's disease is responsible for $50-70 \%$ of all cases, we might conclude there are between 80144 and 112201 patients. According to IMSH-data, 76250 receive anti-dementia drugs and the costs of this kind of medication is $37 \mathrm{M} € /$ year.

Conclusions: As a consequence of the demographic aging, also the number of dementia cases increases. Aparentely, not all Alzheimer's disease patients receive the recommended medication, suggesting this condition is still under-diagnosed. However, figures indicate a positive progression with an increment of treated cases and a reduction of medication-costs.

Keywords: Alzheimer Disease; Cost of Illness; Dementia; Portugal; Prevalence.

\section{INTRODUÇÃO}

A demência constitui a expressão clínica de várias entidades patológicas. A doença de Alzheimer (DA) é a mais prevalente, sendo responsável por 50 a $70 \%$ dos casos. Tanto a incidência como a prevalência da demência aumentam quase exponencialmente com a idade, duplicando aproximadamente a cada 5 anos. ${ }^{1-9}$

A incidência global de demência tem vindo a aumentar drasticamente nas últimas décadas. Se em 2005 se estimava cerca de 7,5/1000 pessoas por ano, ou seja, cerca de um novo caso a cada 7 segundos, ${ }^{10} \mathrm{em} 2012$ as estimativas

1. Centro de Neurociências e Biologia Celular. Universidade de Coimbra. Coimbra. Portugal.

2. Departamento de Neurologia. Faculdade de Medicina. Universidade de Coimbra. Coimbra. Portugal.

3. Consulta de Demência. Serviço de Neurologia. Centro Hospitalar e Universitário de Coimbra. Coimbra. Portugal.

4. Serviço de Reumatologia. Centro Hospitalar do Baixo Vouga. Aveiro. Portugal.

5. Centro de Investigação. Núcleo de Estudos e Intervenção Cognitivo Comportamental (CINEICC). Universidade de Coimbra. Coimbra. Portugal.

6. Departamento de Epidemiologia e Medicina Preventiva. Faculdade de Medicina. Universidade de Coimbra. Coimbra. Portugal.

7. Departamento de Saúde Mental e Psiquiatria. Faculdade de Ciências Médicas. Universidade Nova de Lisboa. Lisboa. Portugal.

8. Programa de Saúde Mental. Direcção Geral de Saúde. Lisboa. Portugal.

Recebido: 24 de Novembro de 2014 - Aceite: 25 de Janeiro de 2015 | Copyright @ Ordem dos Médicos 2015 
apontam para cerca de 7,7/1000 pessoas por ano, o que se traduz em cerca de 1 novo caso a cada 4 segundos. ${ }^{11}$ Relativamente à prevalência, e de acordo com uma meta-análise recente, a prevalência de demência acima dos 60 anos de idade varia entre os $5-7 \%$, sendo mais elevada nos países da América latina (8,5\%) e mais baixa na África sub-sariana (2-4\%). ${ }^{12}$ Assim, em 2010, haveria cerca de 35,6 milhões de pessoas com demência em todo o mundo e as projecções sugerem para que este valor possa duplicar a cada 20 anos, para 65,7 milhões em 2030 e 115,4 milhões em 2050. ${ }^{11,12}$ No entanto, estudos recentes sugerem uma estabilização ou mesmo redução na incidência de demência nos países desenvolvidos, associada à melhoria do estilo de vida e redução das doenças vasculares, o controlo destes fatores de risco levará também a uma redução da mortalidade, pelo que é difícil prever o seu verdadeiro impacto na prevalência da demência. ${ }^{13-16}$

De acordo com a Organização Mundial de Saúde (OMS), em 2004 as demências constituíram a $6^{a}$ principal causa de morte nos países mais desenvolvidos e a DA foi considerada a $5^{\mathrm{a}}$ causa de morte mais frequente em 2006. ${ }^{17,18}$ Contudo, talvez mais relevante do que a mortalidade atribuída à demência seja a morbilidade que lhe está associada. Neste sentido, estima-se que a demência contribua com mais de $11,9 \%$ dos anos vividos com incapacidade nas pessoas acima dos 60 anos, valor superior ao calculado para os acidentes vasculares cerebrais $(9,5 \%)$, para as doenças cardiovasculares $(5,0 \%)$ ou para todas as formas de cancro (2,4\%). ${ }^{11,19}$ Esta elevada morbilidade, associada à cronicidade das demências, acarreta custos directos e indirectos muito elevados, refletidos numa enorme sobrecarga para os sistemas nacionais de saúde, e também com um grande impacto na economia das famílias. ${ }^{20-23}$

Uma vez que a idade é o principal factor de risco para demência, qualquer abordagem epidemiológica neste contexto no nosso país pressupõe um conhecimento das características demográficas nacionais. As tendências demográficas recentes do nosso país são caracterizadas pelo aumento continuado da esperança média de vida ao nascer, a redução da mortalidade infantil, o aumento da emigração, a queda abrupta da fecundidade e o consequente envelhecimento da população. ${ }^{24} \mathrm{O}$ aumento da esperança média de vida ao nascer é um dos fatores mais relevantes que contribui para o agravamento do envelhecimento populacional. Considerando o período 2011-2013, a esperança média de vida à nascença da população residente em Portugal era de 80,0 anos (82,79 anos para as mulheres e 76,91 anos para os homens), tendo aumentado cerca de 3 anos na última década (mais 3,36 anos para os homens e 2,58 anos para as mulheres) considerando as estimativas do triénio $2001-2003$ (73,55 e 80,21 anos para homens e mulheres, respetivamente) ${ }^{24}$. As projecções do Instituto Nacional de Estatística (INE) apontam para que a esperança média de vida à nascença venha a atingir em 2060, para Portugal, os valores de 84,21 anos para homens e 89,88 anos para mulheres. ${ }^{24}$

A proporção de pessoas com 65 ou mais anos dupli- cou nas últimas décadas, passando de $8 \%$ no total da população portuguesa em 1960, para 17\% em 2005 e para $19 \%$ nos censos de 2011 . Este é um reflexo das profundas alterações da estrutura etária da população residente em Portugal, com um decréscimo da população mais jovem e consequente diminuição da base da pirâmide e um aumento da população idosa e respetivo alargamento do topo da pirâmide, configurando-se o cenário de agravamento do envelhecimento da população, uma preocupante realidade atual que apresenta uma tendência crónica. As estimativas de população residente em Portugal dos últimos anos confirmam o duplo envelhecimento demográfico: aumento do número de idosos, diminuição do número de jovens e de pessoas em idade ativa. Em 2001, o índice de enveIhecimento da população era de 102, o que significa que por cada 100 jovens existiam 102 idosos. Apenas dez anos depois, em 2011 o índice de envelhecimento da população passou para 128 e em 2013 verificou-se novo aumento para os 136. Estima-se que a população idosa em Portugal continuará a aumentar nas próximas décadas, atingindo entre $36 \%$ a $43 \%$ da população em 2060 , com um respetivo índice de envelhecimento na ordem dos 287 a 464 idosos por cada 100 jovens. ${ }^{24}$

No nosso país existem poucos estudos publicados dedicados à epidemiologia da demência e não existem dados directos da prevalência das diferentes formas de demência.

Em 1994, foi publicada uma projecção para a população portuguesa da prevalência de demência e de doença de Alzheimer ${ }^{25}$ baseada em dados dos estudos do grupo EURODEM ${ }^{26}$ e nos Censos da população portuguesa de 1991. Garcia e colaboradores extrapolaram, para o ano de 1991, um total de 92470 de indivíduos com demência e 48706 casos de doença de Alzheimer em Portugal, acima dos 30 anos de idade. ${ }^{25}$

Entre 2003 e 2008 foi realizado o primeiro estudo de base populacional para determinação da prevalência de demência e defeito cognitivo ligeiro (DCL) em Portugal. ${ }^{27}$ O estudo abrangeu uma amostra da população com idades compreendidas entre os 55 e os 79 anos de duas comunidades do norte do país. Decorreu em duas fases: uma primeira fase (Fase I) de detecção dos casos através de entrevista e realização de escalas de classificação e de testes neuropsicológicos e uma segunda fase de validação clínica, através de entrevista e exame clínico por neurologista (Fase II). Na Fase I foram encontrados 88 indivíduos que cumpriam critérios para demência. A Fase II, com uma adesão de apenas $56,2 \%$, revelou uma prevalência global de demência, na população entre os 55 e os 79 anos de idade, de $2,7 \%$. A prevalência global de DCL foi de $12,3 \%{ }^{27,28}$

Para além dos dados epidemiológicos obtidos por estudos de base populacional ou por extrapolação a partir de meta-análises europeias, existem diversos indicadores indirectos da prevalência de uma doença ou de um determinado grupo nosológico. São exemplos as informações obtidas a partir dos registos dos médicos de família, dos internamentos hospitalares ou em situações de atendimento urgente, os diagnósticos registados em instituições resi- 
denciais, certificados de óbito e também o consumo de fármacos específicos para uma determinada patologia. ${ }^{29}$ No que se refere à demência, a maioria destas fontes de informação é pouco fiável. Em primeiro lugar porque a demência é uma situação de diagnóstico complexo, que implica recursos pouco acessíveis a algumas populações e que é ainda mal conhecida de muitos agentes de saúde; por estas razões, encontra-se seguramente sub-diagnosticada a nível das estruturas primárias de saúde ou de institucionalização; em segundo lugar, tratando-se de uma situação crónica de alta morbilidade, mas que raramente se constitui como causa directa de morte, estará muito provavelmente sub-identificada nos certificados de óbito. Na verdade, os dados do INE referentes ao ano de 2000 , indicam um total de 616 óbitos por DA, correspondendo a cerca de $41 \%$ dos óbitos causados por doenças do sistema nervoso, nesse ano. ${ }^{30}$ Dados mais recentes, referentes a $2012^{31}$ apontam um registo oficial de 1740 mortes devido a DA, tendo esta causa de morte atingido maioritariamente as mulheres (65\%) e a população idosa (a idade média ao óbito por esta causa foi de 83,1 anos, não se tendo verificado nenhum registo em idades inferiores a 45 anos). Os óbitos por DA representam $1,6 \%$ da mortalidade no país, apresentando uma taxa bruta de mortalidade de 16,6 óbitos por 100000 habitantes e uma taxa de mortalidade padronizada para as idades de 65 e mais anos de 66,7 óbitos por 100000 habitantes.

A informação decorrente da utilização de fármacos específicos é mais interessante e previsivelmente mais fiável, uma vez que os fármacos dirigidos ao tratamento dos sintomas cognitivos só serão utilizados no contexto da demência e essencialmente no tratamento da DA. Poderá assim fornecer uma estimativa aproximada dos diagnósticos, bem como dos custos directos que resultam desta prescrição. ${ }^{29}$

Com o progressivo envelhecimento demográfico, é expectável que o número de casos de demência tenha aumentado nos últimos anos. Pretende-se, portanto, obter uma estimativa mais actualizada da prevalência de demência em Portugal, mediante uma projecção, para a população portuguesa, dos valores de prevalência de demência encontrados para a Europa ocidental pelo estudo de Ferri e colaboradores, ${ }^{10}$ utilizando os dados mais recentes do INE de acordo com as Estimativas de População Residente, à data de 16 de Junho de $2014 .^{32}$
Estes resultados foram comparados com a estimativa de casos identificados no nosso país (diagnósticos efectivos), calculado a partir do consumo de medicamentos específicos para demência. Decorre desta avaliação a estimativa dos gastos inerentes para o Sistema Nacional de Saúde e para os utentes/família, os quais representarão uma das fracções dos custos directos imputáveis à demência.

\section{MATERIAL E MÉTODOS}

Os valores de prevalência de demência ajustada à idade foram calculados a partir do estudo de Ferri e colaboradores, ${ }^{10}$ conduzido pela Alzheimer's Disease International (a metodologia deste estudo é apresentada no Apendix 1). Na nossa investigação, utilizámos as estimativas de prevalência de demência para a região EURO A, onde se inclui o nosso país, e aplicámo-las à população residente em Portugal, de acordo com as Estimativas de População Residente mais recentes, à data de 16 de Junho de 2014, ${ }^{32}$ calculando igualmente os intervalos de confiança a $95 \%$ das prevalências estimadas ${ }^{33}$.

A estimativa dos diagnósticos de DA e encargos financeiros com a medicação específica baseou-se nas informações que nos foram fornecidas pelo Intercontinental Marketing Services Health para os anos 2009-2013. Para o cálculo do número de doentes tratados, indicador dos diagnósticos efectivos, utilizou-se a seguinte fórmula:

$\left[\mathrm{N}^{\circ}\right.$ de Comprimidos $\times$ (Dosagem do comprimidos / Dose Diária recomendada) / 365].

\section{RESULTADOS}

Considerando as Estimativas de População Residente mais recentes, à data de 16 de Junho de $2014^{32}$ que apontam para uma população total de 10427301 pessoas, das quais 2713715 pessoas têm mais de 60 anos, as estimativas de prevalência de demência para a região EURO A aplicadas à população portuguesa indicam que, em 2013 haverá no nosso país aproximadamente 160287 pessoas com demência, correspondendo a $5,91 \%$ da população acima dos 60 anos de idade. Na Tabela 1 indicam-se as estimativas por grupo etário. Pode ver-se que no grupo etário dos 60-64 anos existirão 5795 doentes, mas à medida que avançamos na idade o número de doentes aumenta e, após os 85 anos, serão cerca de 62826.

Tabela 1 - Estimativa da prevalência de demência em Portugal, em 2013

\begin{tabular}{ccccc}
\hline Idade & Prevalência (\%) & IC inf - IC sup & População & N. $^{\circ}$ de doentes \\
\hline $\mathbf{6 0 - 6 4}$ & 0,90 & $0,88-0,92$ & 643872 & 5795 \\
$\mathbf{6 5 - 6 9}$ & 1,53 & $1,47-1,53$ & 575925 & 8639 \\
$\mathbf{7 0 - 7 4}$ & 3,60 & $3,55-3,65$ & 480062 & 17282 \\
$\mathbf{7 5 - 7 9}$ & 6,00 & $5,93-6,07$ & 436117 & 26167 \\
$\mathbf{8 0 - 8 4}$ & 12,20 & $12,09-12,31$ & 324407 & 39578 \\
$\mathbf{8 5 +}$ & 24,80 & $24,63-24,94$ & 253332 & 62826 \\
Total & 5,91 & $5,88-5,93$ & $\mathbf{2 7 1 3 7 1 5}$ & $\mathbf{1 6 0 2 8 7}$ \\
\hline
\end{tabular}




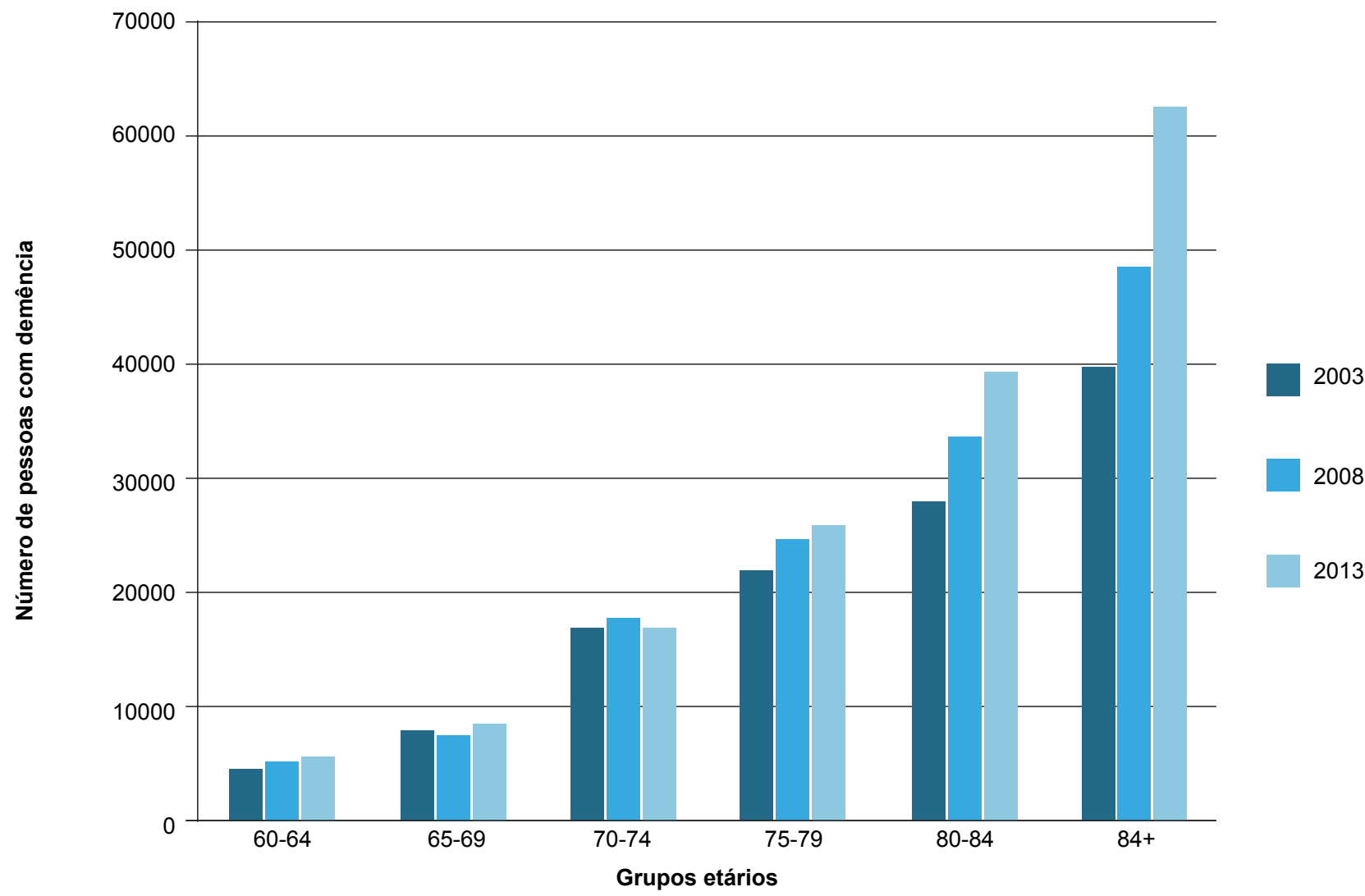

Figura 1 - Perspetiva longitudinal da estimativa do número de pessoas com demência em Portugal

Visando uma perspectiva da evolução longitudinal destas estimativas, aplicamos o mesmo procedimento de cálculo aos valores resultantes das Estimativas de População Residente para os anos de 2003 e de 2008. No ano de 2003 estimava-se existirem 120506 pessoas com demência em Portugal, tendo este valor aumentado para 138390 pessoas em 2008, um acréscimo de 17884 casos no período entre 2003 e 2008, ou seja um aumento médio anual de 3577 casos. Quando comparamos o número estimado de pessoas com demência em 2008 com o estimado para 2013, verificamos um novo acréscimo na ordem dos 21879 casos, ou seja um aumento médio anual de 4377 casos. Os resultados longitudinais por grupo etário são apresentados na Fig. 1.

Partindo do valor obtido de 160287 doentes com demência no nosso país, e sabendo que 50 a $70 \%$ são imputáveis à doença de Alzheimer, poderemos deduzir que em Portugal e em 2013, existiriam entre 80144 e 112201 doentes com DA.

Por outro lado, os dados da IMSH indicam que em 2013 estariam diagnosticados e a receceber anti-demenciais 76250 doentes, representando um encargo financeiro de $37 \mathrm{M} € /$ ano (Tabela 2). Ainda de acordo com as estimativas do IMSH, a evolução tem sido positiva, uma vez que o número de pacientes que recebe tratamento farmacológico para a demência aumentou de 47940 pacientes em 2009 para 76250 pacientes em 2013. Por outro lado, em 2010, registou-se um custo total com este tipo de terapêutica na ordem dos $60 \mathrm{M} € / \mathrm{ano}$, dos quais 22 milhões foram imputa- dos ao SNS e 38 milhões ficaram a cargo dos pacientes (Tabela 2). Não obstante o aumento do número de doses consumidas, sugestivas do aumento do número de pacientes tratados, verificou-se um decréscimo do custo total associado ao consumo de fármacos para a demência, dos cerca de $60 \mathrm{M} € /$ ano registados em 2010 para $37 \mathrm{M} € /$ ano contabilizados em 2013.

\section{DISCUSSÃO}

Dada a inexistência de um estudo populacional português suficientemente abrangente para permitir o conhecimento da prevalência de demência em Portugal, as estimativas europeias constituem, provavelmente, a base mais legítima e mais objectiva de conhecer a situação portuguesa. No estudo de Ferri e colaboradores, ${ }^{10}$ que serviu de base à nossa projecção, utilizou-se o método de Delphi, obtendo-se um consenso sobre a prevalência de demência ajustada à idade para todas as regiões do mundo (definidas pela OMS). O método de Delphi tem como objectivo a obtenção de estimativas quantitativas através da análise qualitativa dos dados. Desta forma podem ser analisados estudos bastante diferentes do ponto de vista metodológico e, quando a informação publicada é escassa, os peritos que constituem o painel de consenso podem fazer inferências usando dados de contextos comparáveis. As estimativas de todos os peritos são agrupadas e entregues, de forma anónima, a cada participante, que revê as suas respostas iniciais face às respostas de todo o grupo. Como o grupo não precisa de se reunir, este método garante o anonima- 
Tabela 2 - Estimativa do número de pacientes e dos encargos financeiros com medicação específica

\begin{tabular}{|c|c|c|c|c|c|}
\hline Ano de referência & 2009 & 2010 & 2011 & 2012 & 2013 \\
\hline $\mathbf{N}^{\circ}$ Pacientes ${ }^{(1)}$ & 46940 & 59499 & 64592 & 68087 & 76250 \\
\hline $\begin{array}{l}\text { Valor Total dos } \\
\text { fármacos }\end{array}$ & $54515763 €$ & $59872287 €$ & $57183996 €$ & $42976801 €$ & $37252264 €$ \\
\hline Estado $^{(2)}$ & $20170832 €$ & $22152746 €$ & $21158079 €$ & $15901416 €$ & $13783338 €$ \\
\hline Paciente & $34344931 €$ & $37719541 €$ & $36025917 €$ & $27075385 €$ & $23468927 €$ \\
\hline
\end{tabular}

do Número de Pacientes: ( ${ }^{\circ}$ de Comprimidos x (Dosagem do comprimidos / Dose Diária recomendada)) / 365.

(2) Foi considerado para o Valor do estado $37 \%$ (escalão C) do Valor total, não é possível com confiança reportar os valores do regime especial.

Nota: Informações do Intercontinental Marketing Services Health (IMSH) - 2013.

to, permitindo a livre expressão de opiniões. ${ }^{10} \mathrm{~A}$ aplicação destas estimativas de consenso à população portuguesa pressupõe que os resultados deste estudo são válidos e que a prevalência de demência no nosso país se comporta de forma semelhante aos restantes países da Europa ocidental. O estudo de Ferri tem algumas limitações, na medida em que, para algumas regiões, os dados epidemiológicos são escassos e, assim, as estimativas obtidas poderão não ser válidas. Contudo, para a região EURO A, na qual se inclui Portugal, existem bastantes estudos com boa qualidade metodológica. Referem-se especificamente os dois estudos efectuados em Espanha, ${ }^{34,35}$ um país culturalmente semelhante ao nosso. Além disso, no painel Delphi o grau de concordância entre os peritos foi excelente logo na primeira ronda, ${ }^{10}$ pelo que é de esperar que a estimativa seja válida.

Verifica-se assim que, em 2013, haveria no nosso país, para a população com mais de 60 anos, aproximadamente 160287 pessoas com demência, o que corresponde a $5,91 \%$ das pessoas abrangidas por estes grupos etários. Recordamos que em 1991 uma estimativa equivalente, calculada a partir dos estudos do grupo EURODEM, propunha uma taxa de $4,6 \%,{ }^{25}$ sugerindo um aumento da prevalência na última década. Admitimos que este aumento na prevalência de demência acima dos 60 anos entre 1991 e 2013 poderá ser explicado pelo aumento da proporção dos muito idosos em Portugal, como sugerido pelos dados estatísticos do INE apresentados. Estes dados são congruentes com a generalidade dos estudos populacionais em que se verifica que a prevalência de demência aumenta de forma quase exponencial com a idade. ${ }^{13-16} \mathrm{De}$ facto, no grupo etário dos 60-64 anos existirão 5795 doentes, mas à medida que avançamos na idade o número de doentes aumenta e, após os 85 anos, serão cerca de 62826. Assim, é entre os grupos etários mais avançados que encontramos o maior número de doentes. O valor obtido de prevalência de $5,91 \%$ é também superior à taxa de $2,7 \%$ sugerida no único estudo de base populacional efectuado em Portugal até à data. ${ }^{27,28}$ Deve no entanto salientar-se que Nunes e colaboradores restringiram o seu estudo a uma faixa populacional entre os 55 e os 79 anos de idade, excluindo muito idosos e, de acordo com a nossa estimativa, é nos grupos etários acima dos 80 anos que se encontram mais de $64 \%$ das pessoas com demência.

Por outro lado, os dados mais recentes do IMSH (2013) indicam que em Portugal, seriam tratadas com fármacos especificamente dirigidos para a vertente cognitiva da demência (inibidores das colinesterases e memantina) 76250 pessoas. A extrapolação destes números para os diagnósticos de DA é aceitável por várias razões: 1) De acordo com a literatura, esta é uma metodologia aplicável a doenças neurodegenerativas e Doença de Alzheimer, embora o estudo sugira uma tendência para uma subestimaçãodo número de diagnósticos, relativamente a avaliações através de índices assistenciais, ${ }^{29}$ 2) esta classe de fármacos tem uma indicação de aplicação relativamente restrita à DA, se excluirmos a sua recomendação de utilização em situações menos relevantes em termos epidemiológicos como a demência com corpos de Lewy e a demência associada à doença de Parkinson; 3) porque a prescrição destas duas classes de fármacos comercializados cobre todo o espectro de severidade da demência, sendo que os inibidores das colinesterases estão indicados nas formas ligeiras e moderadas e a memantina destina-se fundamentalmente às formas severas. De referir, no entanto, que esta nossa estimativa poderá sobre-estimar os doentes com DA efectivamente identificados/tratados, não só porque nesta avaliação estão provavelmente incluídos doentes com outras formas de demência, como também porque as duas classes de fármacos são por vezes utilizadas em associação. Cruzando este resultado de 76250 doentes com os número absoluto de casos de DA sugerido pela estimativa baseada no estudo europeu (80144 e 112201), seremos levados a concluir que esta doença é ainda sub-diagnosticada e sub-tratada. Esta é uma conclusão muito relevante na avaliação da qualidade da assistência prestada no nosso país aos doentes com esta forma de demência, uma vez que a eficácia destas drogas no controlo dos sintomas e na melhoria da qualidade de vida dos doentes e dos seus cuidadores foi repetidamente comprovada em ensaios clínicos robustos, aleatorizados e em dupla ocultação. Além disso estes fármacos são formalmente recomendados para o tratamento da DA em Portugal, conforme consignado na Norma 053/2011 de 27/12/2011 da Direcção Geral da Saúde "Abordagem Diagnóstica e Terapêutica das Alterações Cognitivas (Demências; Doença de Alzheimer)" (http://www. 
dgs.pt/?cr=21530). ${ }^{36}$ Acresce que organismos idóneos, como a Academia Americana de Neurologia, ${ }^{37}$ Federação Europeia das Sociedades de Neurologia ${ }^{38,39}$ são claras na indicação da utilização destes fármacos pelos benefícios comprovados nas várias vertentes sintomáticas da doença. Ainda assim e de acordo com as estimativas apresentadas, a evolução é positiva, uma vez que o número de pacientes que recebe tratamento farmacológico para a demência quase duplicou em 5 anos. Paralelamente verificou-se um decréscimo do custo total associado ao consumo de fármacos específicos para a demência, dos cerca de 60 milhões registados em 2010 para cerca de 37 milhões contabilizados em 2013. Esta redução de custos prende-se provavelmente com a introdução progressiva de genéricos na prescrição nacional. Acrescenta-se que recorrendo a informações do INFARMED - Autoridade Nacional do Medicamento e Produtos de Saúde, I. P. (INFARMED) pudemos verificar que no ano de 2011, 22,19\% dos encargos do Serviço Nacional de Saúde (SNS) destinaram-se a fármacos dirigidos ao Sistema Nervoso Central e que este constituiu o $2^{\circ}$ grupo farmacoterapêutico com maiores encargos, só ultrapassado pelos imputados ao aparelho cardiovascular (29,85\% dos encargos). Três dos fármacos usados na terapêutica da doença de Alzheimer (rivastigmina, donepezilo e memantina) estavam entre as 100 substâncias activas com maiores encargos para o SNS, cada um deles com mais de 100 mil embalagens vendidas em $2011 .{ }^{40}$

$\mathrm{Na}$ avaliação dos encargos financeiros com as doenças, os custos com a medicação incluem-se entre os custos directos (associados ao tratamento, honorários profissionais, exames clínicos, hospitalizações, serviços de terceiros no apoio aos cuidados quotidianos do paciente, entre outros). $\mathrm{Na}$ demência, os denominados custos indirectos (abandono precoce da actividade profissional por parte do doente, diminuição da assiduidade laboral ou mesmo abandono da actividade profissional dos cuidadores informais, aumento da morbilidade nos prestadores de cuidados, entre outros) têm um impacto ainda mais significativo sobre a economia das famílias e o sistema de saúde. ${ }^{20-22}$ As estimativas mundiais dos gastos com demência atingiam os 604 biliões de dólares em 2010. Nos países mais desenvolvidos, $45 \%$ destes gastos eram imputados a cuidados informais, $40 \%$ a cuidados formais e somente $15 \%$ a custos médicos directos). ${ }^{11}$ Por outro lado, os dados apontados por vários estudos indicam uma ampla variação dos encargos financeiros com a demência, os quais se relacionam obviamente com a qualidade dos cuidados de saúde mas também com a precocidade da institucionalização e com a existência de redes de suporte familiares vs. de cuidados formais. ${ }^{41}$ É também consensual nestes estudos a confirmação de uma relação directa entre os gastos e variáveis relacionadas com a gravidade da doença, nomeadamente com a severidade do défice cognitivo, com o grau de incapacidade funcional, a prevalência de alterações psicopatológicas e um maior desgaste dos cuidadores. ${ }^{23}$
Em Portugal são necessários estudos de avaliação objectiva e caracterização da rede global de apoio e dos custos directos e indirectos com a doença. Estes são alguns dos objectivos estratégicos da avaliação promovida pela Direção-Geral da Saúde (DGS), incluídos na "Estruturação de um plano nacional de intervenção em demências". Com efeito, em Maio de 2013 a DGS, através do Programa Nacional para a Saúde Mental (PNSM), promoveu um primeiro seminário com o intuito de iniciar os trabalhos preparatórios desse plano global de intervenção. Para o efeito convidou trinta peritos nacionais, representativos de várias instâncias intervenientes na temática, tendo como dinamizador o Prof. Joël Ménard, Professor Emérito de Saúde Pública da Faculdade de Medicina Paris-Descartes, ex-Diretor Geral de Saúde de França, Presidente da comissão de redação do "Plane Alzheimer France 2008-2012" e Presidente do Conselho Científico Internacional da Fondation pour la Maladie d'Alzheimer et les Maladies Apparentées. No final de 2014 promoveu-se o $2^{\circ}$ seminário, em que foi feito o ponto de situação das iniciativas entretanto promovidas, nomeadamente dos projetos de investigação que o PNSM tem vindo a apoiar financeiramente, tendo em vista a produção de mais evidência científica Portuguesa, que servirá de esteio ao projetado plano nacional de intervenção em demências, que se perspetiva que seja integrado no Programa Nacional para a Saúde das Pessoas Idosas (PNSPI), aprovado por despacho ministerial de 8/6/2004, entretanto descontinuado e que se pretende reativar e articular com o Programa Nacional para a Saúde Mental.

\section{CONCLUSÃO}

O crescimento da população idosa no nosso país parece acompanhar-se de um aumento do número de casos de demência. É expectável que este número continue a aumentar, uma vez que, de acordo com a nossa estimativa, é nos grupos etários acima dos 80 anos que se encontram mais de $64 \%$ das pessoas com demência. Por outro lado, a extrapolação do número de diagnósticos efectivos de DA a partir da sua prescrição específica, sugere que a demência é uma situação ainda sub-diagnosticada. Ainda assim, a evolução tem sido positiva, uma vez que o número de pacientes tratados quase duplicou em 5 anos e os custos com a medicação reduziram quase $50 \%$. Não é demais salientar que o reconhecimento da importância da demência no panorama epidemiológico actual é um ponto de partida obrigatório para a definição das estratégias institucionais de apoio e tratamento dos doentes com demência em Portugal.

\section{AGRADECIMENTOS}

À Consultora IMSH como fonte da informação relativamente ao número de doentes tratados com anti-demenciais e aos custos destes fármacos; a Ana Alberto da Novartis Pharma Portugal, que gentilmente nos referenciou o contacto da IMSH. 


\section{CONFLITOS DE INTERESSE}

Os autores declaram não ter qualquer conflito de interesse relativamente ao presente artigo.

\section{REFERÊNCIAS}

1. Hofman A, Rocca WA, Brayne C, Breteler MB, Clarke M, Cooper B, et al. The prevalence of dementia in Europe: a collaborative study of 1980 1990 findings. Int J Epidemiol. 1991;20:736-48.

2. Jorm AF, Jolley D. The incidence of dementia: a meta-analysis. Neurol. 1998;51:728-33.

3. Fratiglioni L, De Ronchi D, Agüero-Torres $\mathrm{H}$. Worldwide prevalence and incidence of dementia. Drug Aging. 1999;15:365-75.

4. von Strauss E, Viitanen M, De Ronchi D, Winblad B, Fratiglioni L. Aging and the ocurrence of dementia. Arch Neurol. 1999;56:587-92.

5. Fratiglioni L, Launer LJ, Andersen K, Breteler MM, Copeland JR, Dartigues JF, et al. Incidence of dementia and major subtypes in Europe: a collaborative study of population-based cohorts. Neurology. 2000;54:S10-5.

6. Lobo A, Launer LJ, Fratiglioni L, Andersen K, Di Carlo A, Breteler MM, et al. Prevalence of dementia and major subtypes in Europe: a collaborative study of population-based cohorts. Neurology. 2000;54:S4-9.

7. Bermejo-Pareja F, Benito-León J, Vega S, Medrano MJ, Román GC. Incidence and subtypes of dementia in three elderly populations of central Spain. J Neurol Sci. 2008;264:63-72.

8. Corrada MM, Brookmeyer R, Berlau D, Paganini-Hill A, Kawas C. Prevalence of dementia after age 90: results from the $90+$ Study. Neurology. 2008;71:337-43

9. Kawas $\mathrm{CH}$. The oldest old and the $90+$ study. Alzheimers Dement. 2008;4:S56-9.

10. Ferri $C P$, Prince $M$, Brayne $C$, Brodaty $H$, Fratiglioni L, Ganguli M, et al. Global prevalence of dementia: a Delphi consensus study. Lancet. 2005;366:2112-7.

11. World Health Organization. Dementia: a public health priority. Genebra: WHO; 2012.

12. Prince M, Bryce R, Albanese E, Wimo A, Ribeiro W, Ferri CP. The global prevalence of dementia: a systematic review and metaanalysis. Alzheimers Dement. 2013;9:63-75.

13. Wu YT, Matthews FE, Brayne C. Dementia: time trends and policy responses. Maturitas. 2014;79:191-5

14. Matthews FE, Arthur A, Barnes LE, Bond J, Jagger C, Robinson L, et al. A two-decade comparison of prevalence of dementia in individuals aged 65 years and older from three geographical areas of England: results of the Cognitive Function and Ageing Study I and II. Lancet. 2013;382:1405-12.

15. Larson EB, Yaffe K, Langa KM. New insights into the dementia epidemic. N Engl J Med. 2013;369:2275-7.

16. Jacqmin-Gadda $H$, Alperovitch $A$, Montlahuc $C$, Commenges $D$, Leffondre K, Dufouil C, et al. 20-Year prevalence projections for dementia and impact of preventive policy about risk factors. Eur $\mathrm{J}$ Epidemiol. 2013;28:493-502.

17. Federal Interagency Forum on Aging-Related Statistics. Older americans 2010: key indicators of well-being. Washington: Government Printing Office, Federal Interagency Forum on Aging-Related Statistics; 2010.

18. World Health Organization. The top ten causes of death. Genebra: WHO; 2008.

19. World Health Organization. World health report 2003: shaping the future. Genebra: WHO; 2003

20. Bassuk SS, Wypij D, Berkman LF. Cognitive impairment and mortality in the community-dwelling elderly. Am J Epidemiol. 2000;151:676-88.

21. Rice DP, Fillit HM, Max W, Knopman DS, Lloyd JR, Duttagupta S. Prevalence, costs, and treatment of Alzheimer's disease and related dementia: a managed care perspective. Am J Manage Care. 2001;7:80918

\section{FONTES DE FINANCIAMENTO}

Os autores declaram não ter recebido qualquer subsídio relativo ao presente artigo.

22. Lurders SL, Storani MS. Demência: Impacto para a família e a sociedade. In: Netto MP, editor. Gerontologia: A velhice e o envelhecimento em visão globalizada. Rio de Janeiro: Editora Atheneu; 2002. p. 146-59.

23. Handels RL, Wolfs CA, Aalten P, Verhey FR, Severens JL. Determinants of care costs of patients with dementia or cognitive impairment. Alzheimer Dis Assoc Dis. 2013;27:30-6.

24. Instituto Nacional de Estatística. Dia mundial da população. Lisboa: INE; 2014.

25. Garcia C, Costa C, Guerreiro M, Leitão O, Mendonça A, Umbelino J. Estimativa da prevalência da demência e da Doença de Alzheimer em Portugal. Acta Med Port. 1994;7:487-91.

26. Rocca W, Hofman A, Brayne C, Breteler M, Clarke M, Copeland J, et al. Frequency and distribution of Alzheimer's Disease in Europe: a collaborative study of 1980-1990 prevalence findings. Ann Neurol. 1991;30:381-90.

27. Nunes B, Silva RD, Silva MC. Prevalência de defeito cognitivo e demência: resultados de estudo em duas populações do Norte de Portugal. Sinapse. 2008;8:77.

28. Nunes B, Silva RD, Cruz VT, Roriz JM, Pais J, Silva MC. Prevalence and pattern of cognitive impairment in rural and urban populations from Northern Portugal. BMC Neurol. 2010;10:42.

29. Chini F, Pezzotti P, Orzella L, Borgia P, Guasticchi G. Can we use the pharmacy data to estimate the prevalence of chronic condictions? A comparison of multiple data sources. BMC Public Health. 2011:11:688.

30. Instituto Nacional de Estatística. Destaque: Óbitos pela Doença de Alzheimer, 2000. Lisboa: INE; 2001.

31. Instituto Nacional de Estatística. Risco de morrer 2012. Lisboa: INE; 2014

32. Instituto Nacional de Estatística [homepage na Internet]. Lisboa: INE, IP; Portal de Estatísticas Oficiais; [consultado 2014 Ago 26]. Disponível em: http://www.ine.pt

33. Bernard PM, Lapointe C. Mesures statistiques en épidémiologie. Québec: Presses de l'Université du Québec; 1987.

34. Lobo A, Saz P, Marcos G, Dia JL, De-la-Camara C. The prevalence of dementia and depression in the elderly community in a Southern European Population: the Zaragoza Study. Arch Gen Psychiat. 1995;52:497-506.

35. Manubens JM, Martínez-Lage JM, Lacruz F, Muruzabal J, Larumbe R, Guarch C, et al. Prevalence of Alzheimer's Disease and other dementing disorders in Pamplona, Spain. Neuroepidemiol. 1995;14:155-64.

36. Direção Geral da Saúde. Abordagem terapêutica das alterações cognitivas. Lisboa: Ministério da Saúde; 2011 [consultado 2014 Set 2]. Disponivel em: http://www.dgs.pt/?cr=21530.

37. Doody RS, Stevens JC, Beck C, Dubinski RM, Kaye JA, Gwyther L, et al. Practice parameter: management of dementia (an evidence-based review). Report of the Quality Standards Subcommittee of the American Academy of Neurology. Neurology. 2001;56:1154-66.

38. Horta JT, Brienb O, Gainottic G, Pirttilad T, Popescue BO, Rektorovaf I, et al. EFNS guidelines for the diagnosis and management of Alzheimer's disease. Eur J Neurol. 2010;17:1236-48.

39. Waldemar G, Dubois B, Emre M, Georges J, McKeith IG, Rossor M, et al. Recommendations for the diagnosis and management of Alzheimer's disease and other disorders associated with dementia: EFNS guideline. Eur J Neurol. 2007;14:1-26

40. INFARMED. Estatística do Medicamento 2011 Lisboa: Autoridade Nacional do Medicamento e Produtos de Saúde, IP; 2011.

41. Olesen J, Gustavsson A, Svensson M, Wittchen HU, Jönsson B. The economic cost of brain disorders in Europe. Eur J Neurol. 2012;19:15562. 
Isabel SANTANA, Filipa FARINHA, Sandra FREITAS, Vítor RODRIGUES, Álvaro CARVALHO

\section{Epidemiologia da Demência e da Doença de Alzheimer em Portugal: Estimativas da Prevalência e dos Encargos Financeiros com a Medicação}

Acta Med Port 2015:28:182-188

Publicado pela Acta Médica Portuguesa, a Revista Científica da Ordem dos Médicos

Av. Almirante Gago Coutinho, 151

1749-084 Lisboa, Portugal.

Tel: +351218428 215

E-mail: submissao@actamedicaportuguesa.com

www.actamedicaportuguesa.com

ISSN:0870-399X | e-ISSN: 1646-0758

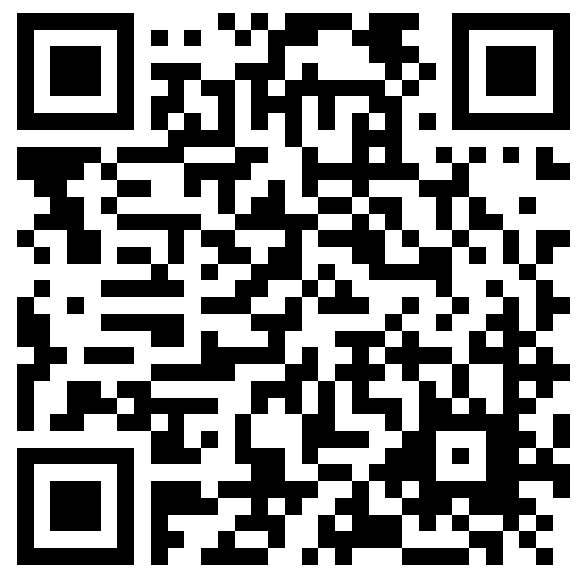

